\title{
Atención sostenida en adultos universitarios: Evidencia de validez de constructo del
}

\section{Test d2}

\section{Josiane Pawlowski}

\section{Universidad de Iberoamerica}

\section{Resumen}

Se evaluó la atención sostenida de 32 universitarios con diagnóstico de déficit de atención pareados a 64 controles por las variables sexo, edad, tiempo de estudio y carrera. Se les aplicó un cuestionario y el Test de Atención d2, de manera colectiva o individual en aproximadamente 30 minutos, incluyendo las explicaciones de la investigación y la concordancia de los participantes mediante firma del consentimiento informado. Los datos fueron analizados con las pruebas $t$ de Student o $U$ de Mann-Whitney. Los resultados indicaron diferencia significativa $(p<.01)$ en la comparación de medias entre las dos muestras para las medidas TR, TA, TOT y CON del test d2, lo que no fue observado para $\mathrm{O}$, C y VAR. Los participantes con déficit de atención presentaron resultados más bajos en la velocidad del procesamiento (TR), la precisión del procesamiento (TA), el control atencional e inhibitorio (TOT), y la concentración (CON). Esos resultados indican una evidencia de validez de constructo del test de atención d2 para las variables TR, TA, TOT y CON de la prueba.

Palabras clave: atención sostenida, déficit de atención, Test d2, universitarios, validez

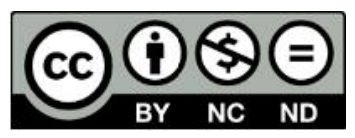




\section{Introducción}

La atención es una función compleja que interrelaciona con diversos procesos cognitivos (Lezak, Howieson, \& Loring, 2004). De esa manera, no es reducida a una simple definición. Para Parasuraman (1998), la atención puede ser considerada un constructo multidimensional en que se diferencian tres componentes principales: selección, vigilancia y control. La atención selectiva permite a los organismos extraer información relevante mientras ignoran los estímulos distractores (Gomez-Ramirez, Hysaj, \& Niebur, 2016). La atención sostenida se define por la capacidad de asignar recursos de procesamiento durante algún tiempo a una demanda de tareas específicas, mientras se ignoran nuevos estímulos que también demandan atención (Schweizer, 2005). El control inhibitorio se refiere a la capacidad de inhibir intencionalmente las respuestas motoras de acuerdo con el cambio de claves contextuales (Nigg, 2000).

La atención es fundamental para la administración de diferentes actividades y para la ejecución de las tareas diarias en nuestra vida. De esa manera, un déficit atencional puede disminuir el rendimiento y traer algunos problemas en el ámbito escolar o laboral de los adultos, por ejemplo. Según Kates (2005), algunos de los indicios de la presencia del déficit de atención en los adultos incluyen la inestabilidad en el trabajo y en las relaciones, así como la desorganización o problemas para completar las tareas, problemas escolares, síntomas de inquietud, cambios de humor o problemas de concentración. Ese autor indica también que el trastorno por déficit de atención es prevalente pero muchas veces no es reconocido en los adultos o es de difícil diagnóstico. Eso sucede porque el diagnóstico debe incluir el inicio de

\section{py:


los síntomas antes de los siete años, información que no es fácil de rescatarse en el momento del diagnóstico (Kates, 2005).

Por ser una función compleja y un constructo multidimensional, para la evaluación de los diferentes tipos de atención existen tareas, pruebas y paradigmas diversos (EstévezGonzález, García-Sánchez, \& Junqué, 1997). Entre esos diferentes tipos, la atención sostenida puede ser evaluada por el Test de Atención d2 (Brickenkamp, 2002). Ese instrumento es conocido en Alemania como test de concentración o test de atención selectiva, y en Estados Unidos recibe la denominación de test de amplitud atencional, de atención selectiva o de atención sostenida (Lezak, 1995). De los constructos que el test d2 examina, Davis y Zillmer (1999) destacan asignación de la atención, velocidad de procesamiento, cantidad de trabajo realizado, motivación, control inhibitorio, cumplimiento de la regla, esmero, velocidad, precisión de su rendimiento y cantidad de trabajo realizado después de la corrección de errores.

La prueba de atención d2 es una medida que puede ser útil a la evaluación neuropsicológica (Brickenkamp, 2002). Las investigaciones que utilizan el test d2 son realizadas en diferentes tipos de muestras: niños, adolescentes y adultos. Wassenberg et al. (2008) investigaron el desarrollo de la falta de atención, la impulsividad y la velocidad de procesamiento, medida por la prueba $\mathrm{d} 2$, en un estudio transversal en niños de siete a trece años. Freitas y Aguiar (2012) evaluaron, además de la memoria y la percepción, la atención medida por el test d2 en pacientes con esclerosis múltiple de 20 a 55 años de edad. CléryMelin y Gorwood (2017) evaluaron la atención de pacientes deprimidos por medio de la 
prueba d2 con el objetivo de investigar el papel del déficit de atención como marcador de pronóstico después de un episodio depresivo mayor (MDE).

A respecto de las investigaciones de las condiciones psicométricas del test, Davis y Zillmer (1999) administraron el test d2 y subpruebas de la Escala Wechsler de Inteligencia para Adultos Revisada - WAIS-R a una muestra de 506 estudiantes universitarios estadunidenses de 18 a 32 años de edad. Los resultados de esta investigación proporcionaron evidencias de la validez discriminante del test d2. Zillmer y Kennedy (1999) investigaron la validez de constructo del test d2 en la muestra de 506 universitarios. La evaluación incluyó, además del test d2, la administración del Stroop Test, de las formas A y B del Trail Making Test y el Symbol Digit Modalities Test (SDMT). La investigación señaló la sensibilidad del test d2 para evaluar atención y concentración en la muestra de Estados Unidos. Bates y Lemay Jr. (2004) examinaron la consistencia interna y la validez convergente y discriminante de la prueba d2 en una muestra de 364 adultos estadounidenses. Los resultados señalaron que el d2 presenta consistencia interna y es una medida válida de la precisión y velocidad de la exploración visual.

Investigaciones más actuales con el test d2 buscan ofrecer datos normativos para poblaciones específicas y también proporcionan guías prácticos para puntajes de la prueba. Por ejemplo, Jiménez et al. (2012) analizaron el desarrollo evolutivo de la atención y presentaron datos normativos de la prueba de atención d2 en escolares con edades entre los 6 años y 3 meses y los 12 años y 4 meses, manifestando por medio de ese estudio la existencia de cambios significativos en las variables de productividad y efectividad. Steinborn, Langner,

\section{pyseon


Flehmig y Huestegge (2017) presentaron un análisis psicométrico de los índices de desempeño comúnmente utilizados de la prueba de atención sostenida d2, y ofrecen recomendaciones del uso basadas en su investigación.

Asumiendo que el Test d2 es una medida útil a la evaluación neuropsicológica, que las alteraciones en la atención y en la concentración son frecuentemente asociadas a daño neurológico (Lezak, Howieson, \& Loring, 2004) y traen problemas en el ámbito escolar o laboral de los adultos, esa investigación pretende contribuir al incremento del área de evaluación neuropsicológica en Costa Rica. Para eso, el objetivo es investigar y demonstrar evidencias de la validez de la prueba de atención d2 en una muestra de universitarios con relato de diagnóstico de déficit de atención comparada a participantes sin déficit de atención.

\section{Método}

\section{Participantes}

Fueron evaluados a 32 estudiantes universitarios que indicaron la presencia de diagnóstico de déficit de atención mediante un instrumento de auto reporte. Esos participantes fueron comparados a una muestra de 64 estudiantes universitarios que informaron la ausencia de déficit atencional, pareados a los primeros por sexo, nivel de escolaridad, carrera que estudiaban, edad y tiempo de estudio, con oscilaciones en algunos casos de un año para más o para menos en las dos últimas variables. Los participantes eran costarricenses o extranjeros con residencia permanente en Costa Rica (dos en la muestra con déficit de atención, nacidos en Suiza y Puerto Rico, y tres entre los participantes controles, 
dos de México y uno de Colombia). Las demás características de la muestra son presentadas en la Tabla 1.

Tabla 1. Características de la muestra

\begin{tabular}{lcc}
\hline Participantes & $\begin{array}{c}\text { Con déficit de atención } \\
(n=32)\end{array}$ & $\begin{array}{c}\text { Sin déficit de atención } \\
(n=64)\end{array}$ \\
\hline Sexo & 16 & 32 \\
F & 16 & 32 \\
M & & \\
Edad & $23.44(5.99)$ & $23.44(5.90)$ \\
$M(D S)$ & $13.71(1.57)$ & $14.05(2.02)$ \\
Tiempo de estudio (años) & & \\
$M(D S)$ & $40.6 \%$ & $42.2 \%$ \\
Universidad & $59.4 \%$ & $57.8 \%$ \\
Pública & & 46 \\
Privada & 23 & 14 \\
Carrera & 7 & 4 \\
$\quad$ Psicología & 2 & \\
Física & & \\
Ingeniería &
\end{tabular}

\section{Instrumentos}

Fueron aplicados los siguientes instrumentos de medida:

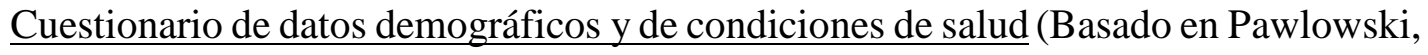
2011): incluye cuestiones sobre datos personales, académicos y médicos.

Test de Atención d2 (Brickenkamp, 2002): Es un test de tiempo limitado para medir la atención sostenida, la atención selectiva y la concentración mental, entendida como la capacidad de atender selectivamente, de forma rápida y precisa, a ciertos aspectos relevantes de una tarea mientras se ignoran los irrelevantes. El test se puede administrar de forma

\section{py e.


individual o colectiva con una duración entre 8 y 10 minutos. La prueba presenta 14 líneas con 47 caracteres, totalizando 658 elementos. Esos estímulos contienen las letras "d" o "p" que pueden estar acompañados de una o dos pequeñas líneas situadas, individualmente o en pareja, en la parte superior o inferior de cada letra. La tarea del sujeto consiste en revisar atentamente, de izquierda a derecha, el contenido de cada línea y marcar toda letra "d" que tenga dos pequeñas rayitas (las dos arriba, las dos debajo o una arriba y otra debajo). En el Manual estos elementos (es decir, los estímulos correctos) son presentados como elementos relevantes. Las demás combinaciones (las "p" con o sin rayitas y las “d” con o ninguna rayita) se consideran "irrelevantes" y no deberían ser marcadas. En cada línea el sujeto dispone de 20 segundos. Las puntuaciones resultantes del Test d2 son presentadas en la Tabla 2. En la mayoría de los estudios las puntuaciones del d2 son muy fiables $(r>0,90)$, independientemente del estadístico empleado; incluso en las muestras especiales los índices se encuentran entre 0,70 y 0,90 (Brickenkamp, 2002). Los estudios de validez en relación con otras pruebas de atención y concentración han demostrado buenos resultados. Las correlaciones con otros testes de atención y concentración se encuentran entre 0,31 a 0,71 , dependiendo de la muestra, la medida de comparación, y el escore utilizado para los análisis estadísticos (Strauss, Sherman, \& Spreen, 2006). 
Tabla 2. Puntuaciones resultantes del Test de Atención d2*

\begin{tabular}{|c|c|c|}
\hline Puntuación & Descripción & ¿Qué mide? \\
\hline $\begin{array}{l}\text { TR: Total de } \\
\text { Respuestas }\end{array}$ & $\begin{array}{l}\text { Número de elementos } \\
\text { intentados en las } 14 \text { líneas, } \\
\text { tanto los relevantes como los } \\
\text { irrelevantes }\end{array}$ & $\begin{array}{l}\text { Velocidad de procesamiento, } \\
\text { cantidad de trabajo realizado y } \\
\text { motivación }\end{array}$ \\
\hline $\begin{array}{l}\text { TA: Total } \\
\text { Aciertos }\end{array}$ & $\begin{array}{l}\text { Número de elementos } \\
\text { relevantes correctos }\end{array}$ & $\begin{array}{l}\text { Cantidad de trabajo y precisión del } \\
\text { procesamiento }\end{array}$ \\
\hline O: Omisiones & $\begin{array}{l}\text { Número de elementos } \\
\text { relevantes intentados pero } \\
\text { no marcados }\end{array}$ & $\begin{array}{l}\text { Control atencional, cumplimento de } \\
\text { una regla, precisión de la búsqueda } \\
\text { visual y calidad de la actuación }\end{array}$ \\
\hline C: Comisiones & $\begin{array}{l}\text { Número de elementos } \\
\text { irrelevantes marcados }\end{array}$ & $\begin{array}{l}\text { Control inhibitorio, cumplimento de } \\
\text { una regla, precisión del } \\
\text { procesamiento, minuciosidad y } \\
\text { flexibilidad cognitiva }\end{array}$ \\
\hline $\begin{array}{l}\text { TOT: Efectividad } \\
\text { total en la prueba }\end{array}$ & $\begin{array}{l}\text { Número de elementos } \\
\text { procesados menos el número } \\
\text { total de errores cometidos: } \\
\mathrm{TR}-(\mathrm{O}+\mathrm{C})\end{array}$ & $\begin{array}{l}\text { Control atencional e inhibitorio y } \\
\text { relación entre la velocidad y } \\
\text { precisión }\end{array}$ \\
\hline $\begin{array}{l}\text { CON: Indice } \\
\text { concentración }\end{array}$ & $\begin{array}{l}\text { Número de elementos } \\
\text { relevantes marcados menos } \\
\text { el número de comisiones: } \\
\text { TA-C }\end{array}$ & $\begin{array}{l}\text { Índice de concentración o del } \\
\text { equilibrio entre velocidad y } \\
\text { precisión de la actuación }\end{array}$ \\
\hline $\begin{array}{l}\text { VAR: Índice } \\
\text { variación }\end{array}$ & $\begin{array}{l}\text { Diferencia entre la mayor y } \\
\text { la menor productividad: } \\
(\mathrm{TR}+)-(\mathrm{TR}-)^{1}\end{array}$ & $\begin{array}{l}\text { Estabilidad y consistencia en el } \\
\text { tiempo, variación o fluctuación en el } \\
\text { modo de trabajar }\end{array}$ \\
\hline
\end{tabular}

\section{Tipo de estudio y procedimientos}

Esa investigación es del tipo cuantitativo, transversal y correlacional (Hernández, Fernández, \& Baptista, 2006). Fueron realizadas evaluaciones colectivas o individuales con duración aproximada de 30 minutos, incluyendo las explicaciones de la investigación y la

\section{pes $\cos _{\ln }$


concordancia de los participantes mediante firma del consentimiento informado. Todos los participantes firmaron el consentimiento informado que expuso los objetivos de la investigación, riesgos, beneficios y en que se aclaró el derecho al resguardo de los datos, anonimato y confidencialidad de la información fornecida. Una comisión científica de profesores de UNIBE evaluó la pertinencia del estudio. La recolección de los datos fue conducida por una psicóloga capacitada en la aplicación de las pruebas y se realizó en aulas de las universidades o en espacios de estudios con la menor cantidad de elementos distractores posible.

\section{Análisis de datos}

El análisis de datos se llevó a cabo con el SPSS 19 (Statistical Package for Social Sciences). Fue realizada la prueba de normalidad Kolmogorov-Smirnov para verificar la distribución de las variables del Test d2. Los datos fueron analizados con las pruebas $t$ de Student o U de Mann-Whitney dependiendo del resultado de la distribución de las variables.

\section{Resultados}

La prueba de normalidad Kolmogorov-Smirnov indicó que las variables del test d2: TR, TA, TOT y CON presentan distribución en esta muestra que se acerca a la normal, mientras las demás (O, C y VAR) no presentan esta condición. Debido a eso, se muestran en la Tabla 3 los resultados de los análisis de comparación entre las muestras con el test $t$ de 
Student para las variables con distribución normal y en la Tabla 4 los resultados de comparación mediante el test non paramétrico $U$ de Mann-Whitney para las demás variables.

Tabla 3. Medias, desviaciones estándar y resultados de la prueba $t$ de Student para las medidas TR, TA, TOT y CON del Test d2 evaluadas en universitarios con déficit de atención (DA) y controles

\begin{tabular}{|c|c|c|c|c|c|c|c|}
\hline \multirow[b]{2}{*}{ Medidas del d2 } & \multicolumn{2}{|c|}{$\begin{array}{l}\text { Con DA } \\
(n=32)\end{array}$} & \multicolumn{2}{|c|}{$\begin{array}{c}\text { Controles } \\
(n=64)\end{array}$} & \multirow[b]{2}{*}{$t$} & \multirow[b]{2}{*}{$g l$} & \multirow[b]{2}{*}{$p$} \\
\hline & $M$ & $D S$ & $M$ & $D S$ & & & \\
\hline TR & 477.03 & 71.29 & 526.38 & 72.09 & 3.17 & 94 & .002 \\
\hline TA & 178.91 & 32.23 & 201.19 & 37.67 & 2.86 & 94 & .005 \\
\hline TOT & 452.88 & 64.40 & 499.27 & 69.88 & 3.15 & 94 & .002 \\
\hline $\mathrm{CON}$ & 178.44 & 32.52 & 200.16 & 38.38 & 2.75 & 94 & .007 \\
\hline
\end{tabular}

Como se puede verificar en la Tabla 3, los análisis indican diferencia significativa ( $p$ $<.01)$ en la comparación de medias entre las dos muestras para las cuatro variables estudiadas, observándose que los participantes con déficit de atención presentan resultados más bajos en todas las variables. 
Tabla 4. Resultados de la prueba Mann-Whitney U para las medidas $O, C$ y VAR del Test d2 de los participantes con Déficit de Atención (DA) y controles

\begin{tabular}{|c|c|c|c|c|c|}
\hline \multirow[b]{2}{*}{$\begin{array}{l}\text { Participantes } \\
\text { Medidas del Test d2 }\end{array}$} & \multicolumn{2}{|c|}{ Rango promedio } & \multirow[t]{2}{*}{$U$} & \multirow[t]{2}{*}{$z$} & \multirow[t]{2}{*}{$p$} \\
\hline & $\begin{array}{l}\text { Con DA } \\
(n=32)\end{array}$ & $\begin{array}{c}\text { Controles } \\
(n=64)\end{array}$ & & & \\
\hline $\mathrm{O}$ & 47.22 & 49.14 & 983.00 & -.319 & .75 \\
\hline $\mathrm{C}$ & 49.19 & 48.16 & 1002.00 & -.214 & .83 \\
\hline VAR & 52.03 & 46.73 & 911.00 & -.881 & .38 \\
\hline
\end{tabular}

En la Tabla 4 se puede observar que los participantes con déficit de atención no presentan diferencia significativa en los resultados de las variables $\mathrm{O}, \mathrm{C}$ y VAR de la prueba d2 cuando comparados a los controles.

\section{Discusión}

Ese estudio presentó el objetivo de investigar y demonstrar una evidencia de la validez de la prueba de atención d2 en una muestra de universitarios con diagnóstico de déficit de atención informado mediante auto reporte comparada a participantes que no presentaban este déficit. Esta comparación indicó diferencias entre esas muestras para algunas de las medidas del Test d2.

Los resultados indicaron que los participantes con déficit atencional muestran menor desempeño en la prueba, lo que es observado por los resultados significativamente más bajos en la velocidad del procesamiento, la cantidad de trabajo realizado y la motivación (TR), la precisión del procesamiento (TA), el control atencional e inhibitorio y la relación entre la velocidad y precisión (TOT), y la concentración o el equilibrio entre velocidad y precisión 
de la actuación (CON). De estas medidas, el TR y CON suelen ser muy fiables y la medida del TOT es la principal para la validación del test y la más utilizada en los estudios experimentales y aplicados, según la versión del manual de Brickenkamp (2002), traducida al castellano por Seisdedos.

De manera diferente, las demás variables del test analizadas en esa investigación $(\mathrm{O}$, C y VAR) no presentaron diferencia significativa en la comparación entre los participantes con y sin déficit de atención. La cantidad de errores en las dos muestras fue muy semejante, lo que indica que $\mathrm{O}$ y $\mathrm{C}$ no son medidas cuantitativas fiables para discriminar el control atencional o el control inhibitorio entre personas que presentan déficit de atención de aquellas que no lo presentan. La medida de VAR, aunque haya sido más alta para personas con déficit de atención, no presentó diferencia estadísticamente significativa cuando comparada a personas que no presentaban este déficit. La puntuación VAR extremadamente alta puede sugerir inconsistencia en el trabajo o falta de motivación. Según Brickenkamp (2002), las medidas de $\mathrm{O}, \mathrm{C}$ y VAR en general no se distribuyen normalmente y son muy poco fiables. En una investigación bastante actual sobre la puntuación de rendimiento en la prueba d2, Steinborn et al. (2017) presentan algunas directrices prácticas sobre el uso del test y recomiendan que las variables que miden "aspectos de la precisión del desempeño (es decir, errores de omisión y comisión) y la variabilidad podrían servir como aspectos secundarios además de la velocidad de ejecución, aunque se recomienda precaución con respecto a las interpretaciones" (p. 13).

\section{pyes.o.


Considerando los resultados obtenidos, la presente investigación permitió evidenciar diferencias entre participantes con déficit de atención de aquellos que no presentaban este déficit en cuatro medidas del Test de Atención d2. Este resultado indica una evidencia de que las medidas TR, TA, TOT y CON del Test de Atención d2 presentan validez de constructo obtenida mediante el método de grupos contrastados (Cohen, \& Swerdlik, 2006).

Si bien esa evidencia fue encontrada, es importante considerar que otros estudios de evidencias de validez deban ser conducidos, así como estudios sobre métodos de puntuación de los resultados. En ese sentido, por ejemplo, al investigar los efectos experimentales del retest sobre la velocidad y precisión del rendimiento (errores de omisión y comisión), Steinborn et al. (2017) sugieren a los profesionales que trabajan en contextos clínicos y neuropsicológicos que "la velocidad de rendimiento (o la velocidad de error corregida, respectivamente) sea la única medida elegible para una evaluación altamente confiable, y por lo tanto debe considerarse como el índice de rendimiento primario" (p. 13). Jiménez et al. (2012) también demostraron la existencia de cambios significativos con el paso de los años en las variables de productividad y efectividad, y no en la medida de errores cuando analizaron el desarrollo evolutivo de la atención en escolares con edades entre los 6 años a $\operatorname{los} 12$ años.

A pesar de la relevancia de los resultados de velocidad confirmada por el presente estudio, la utilidad de la medida de los errores no debe ser descartada, pues en otras muestras u otros tipos de estudios puede presentarse importante. Por ejemplo, Cléry-Melin y Gorwood (2017) evaluaron la atención de pacientes deprimidos por medio de la prueba d2 e 
demostraron el papel del déficit de atención como un marcador de pronóstico después de un episodio depresivo mayor (MDE). Con este estudio, los autores concluyeron que el número de errores de omisión de la prueba de atención d2 fue un marcador estable y predictivo para la remisión clínica más funcional.

La relevancia del presente estudio fue indicar la utilidad y demonstrar una evidencia de validez del Test de Atención d2 para la evaluación neuropsicológica de adultos en Costa Rica. Las limitaciones de esta investigación incluyen el uso del instrumento de auto reporte (cuestionario) para detectar la presencia o ausencia del déficit de atención en los participantes cuando una evaluación diagnóstica traería más precisión para la caracterización de los participantes. Además de eso, a pesar de los resultados estadísticamente significativos encontrados, un número más elevado de participantes podría generar hallazgos aún más confiables. Se sugiere el seguimiento de los estudios con otras muestras, así como utilizando otros diseños de investigación o métodos de evaluación y de análisis de los resultados con el objetivo de demonstrar otras evidencias de la validez del Test de Atención d2 para la evaluación de la población costarricense.

\section{Referencias}

Bates, M. E., \& Lemay Jr., E. P. (2004). The d2 Test of Attention: Construct validity and extensions in scoring techniques. Journal of the International Neuropsychological Society, 10(3), 392-400. doi:10.1017/S135561770410307X.

\section{pyes.o.


Brickenkamp, R. (2002). D2, Test de atención (trad. al castellano por n. Seisdedos). Madrid: TEA Ediciones.

Cléry-Melin, M.-L., \& Gorwood, P. (2017), A simple attention test in the acute phase of a major depressive episode is predictive of later functional remission. Depression and Anxiety, 34(2), 159-170. doi:10.1002/da.22575

Cohen, R. J., \& Swerdlik, M. (2006). Pruebas y evaluación psicológica: Introducción a las pruebas y a la medición. (4a.ed.). México: Mc Graw-Hill.

Davis, K. L., \& Zillmer, E. A. (1999). Contrasts between the d2 Test of Attention and intelligence measures from a normative sample. Archives of Clinical Neuropsychology 14(1), 72. doi: 10.1093/arclin/14.1.72.

Estévez-González, A., García-Sánchez, C., \& Junqué, C. (1997). La atención: Una compleja función cerebral. Revista de Neurología, 25(148), 1989-1997.

Freitas, J. O. F., \& Aguiar, C. R. R. A. (2012). Avaliação das funções cognitivas de atenção, memória e percepção em pacientes com esclerose múltipla. Psicologia: Reflexão e Crítica, 25(3), 457-466. doi. 10.1590/S0102-79722012000300005

Gomez-Ramirez, M., Hysaj, K., \& Niebur, E. (2016). Neural mechanisms of selective attention in the somatosensory system. Journal of Neurophysiology, 116(3) 1218-1231. doi: $10.1152 /$ jn.00637.2015

Hernández, R., Fernández, C., \& Baptista, P. (2010). Metodología de la investigación. (5ta ed.). México: McGrawHill. 
Jiménez, J. E., Hernández, S., García, E., Díaz, A., Rodríguez, C., \& Martín, R. (2012). Test de atención D2: Datos normativos y desarrollo evolutivo de la atención en educación primaria. European Journal of Education and Psychology, 5(1), 93-106. Recuperado de http://www.redalyc.org/articulo.oa?id=129324775008

Kates, N. (2005). Attention deficit disorder in adults: Management in primary care. Canadian Family Physician, $\quad$ 51(1), 53-59. Recuperado de https://www.ncbi.nlm.nih.gov/pmc/articles/PMC1479568/pdf/jCFP_v051_pg53.pdf

Lezak, M. D. (1995). Neuropsychological Assessment (3 ${ }^{\mathrm{a}}$ Ed.). Oxford: Oxford University Press. (Original work published 1976).

Lezak, M. D., Howieson, D. B., \& Loring, D. W. (2004). Neuropsychological Assessment. New York: Oxford Univertsity Press.

Nigg, J. T. (2000). On inhibition/disinhibition in developmental psychopathology: Views from cognitive and personality psychology and working inhibition taxonomy. Psychological Bulletin, 126, 220-246. doi: 10.1037/0033-2909.126.2.220

Parasuraman, R. (1998). Th e attentive brain: Issues and prospects . In R. Parasuraman (ed.), The Attentive Brain (pp. 3-15). Cambridge, Mass.: MIT Press.

Pawlowski, J. (2011). Instrumento de Avaliação Neuropsicológica Breve Neupsilin: Evidências de Validade de Construto e de Validade Incremental à Avaliação Neurológica. Tesis de Doctorado, Programa de Pós-Graduação em Psicologia, Instituto de Psicologia, Universidade Federal do Rio Grande do Sul. Porto Alegre, RS.

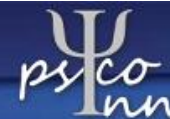


Recuperado

de

http://www.lume.ufrgs.br/bitstream/handle/10183/27835/000765892.pdf

Schweizer, K. (2005). An overview of research into the cognitive basis of intelligence. Journal of IndividualDifferences, 26, 43-51. doi: 10.1027/1614-0001.26.1.43

Steinborn, M. B., Langner, R., Flehmig, H. C., \& Huestegge, L. (2017, April 13). Methodology of Performance Scoring in the d2 Sustained-Attention Test: CumulativeReliability Functions and Practical Guidelines. Psychological Assessment. Advance online publication. doi: 10.1037/pas0000482

Strauss, E., Sherman, E. M. S., \& Spreen, O. (2006). A Compendium of Neuropsychological Tests: Administration, Norms and Commentary. New York: Oxford University Press.

Wassenberg, R., Hendriksen, J. G. M., Hurks, P. P. M., Feron, F. J. M., Keulers, E. H. H., Vles, J. S. H., \& Jolles, J. (2008). Development of inattention, impulsivity, and processing speed as measured by the $\mathrm{d} 2$ test: results of a large cross-sectional study in children aged 7-13. Child Neuropsychology, 14(3), 195-210. doi. $10.1080 / 09297040601187940$

Zillmer, E. A., \& Kennedy, C. H. (1999). Construct validity for the d2 Test of Attention. Archives of Clinical Neuropsychology, 8, 728. Paper presented at the 19th Annual Meeting of the National Academy of Neuropsychology, San Antonio, TX, November, 1999. 